\title{
Oesophagus perforation mimicking an acute chest pain syndrome
}

\author{
Giovambattista Desideri · Giuseppe Limongelli • \\ Ezio De Pratti
}

Received: 10 February 2015/ Accepted: 15 February 2015/Published online: 12 March 2015

(C) SIMI 2015

\section{Clinical case}

A 71-year-old patient was admitted to the emergency department (ED) of a local hospital complaining of mild abdominal pain with an onset the night before. He was a heavy smoker for 25 years, and had a history of hypertension and dyslipidemia for 18 years. He also suffered from stage 2 renal failure over prior 9 years. Physical examination showed a mild-to-moderate abdominal pain on deep palpation, while chest and heart examinations did not reveal any relevant clinical findings (blood pressure 120/70 $\mathrm{mmHg}$, heart rate $72 \mathrm{bpm}$ ). An abdominal echography showed gallstones. The ECG revealed non-specific repolarization abnormalities (Fig. 1a). Echocardiography revealed only a mild dilatation of the ascending aorta (43 mm) (Fig. 1b). Laboratory tests were all within the reference ranges with the exception of a mild increase in the serum creatinine $(1.2 \mathrm{mg} / \mathrm{dL})$. Physicians advised the patient to remain under observation in the hospital, but he decided to return to his home. 3 days later, he returned to the ED for chest pain and dyspnoea. Physical examination showed tachypnea, and hypotension (blood pressure

\author{
G. Desideri $(\bowtie)$ \\ Department of Life, Health and Environmental Sciences, \\ University of L'Aquila and Geriatric Unit, Avezzano Hospital, \\ L'Aquila, Italy \\ e-mail: giovambattista.desideri@cc.univaq.it \\ G. Desideri \\ Geriatric Unit, Avezzano Hospital, L’Aquila, Italy \\ G. Limongelli \\ Monaldi Hospital, Second University of Naples, Naples, Italy \\ E. De Pratti \\ Emergency Unit, Avezzano Hospital, L’Aquila, Italy
}

$75 / 55 \mathrm{mmHg}$, heart rate $75 \mathrm{bpm}$ ), a S3 gallop and basal pulmonary rales. Arterial blood gas analysis revealed a compensated metabolic acidosis (anion gap $16.2 \mathrm{mmol} / \mathrm{L}$ ). The ECG revealed ST elevations in the inferolateral leads (Fig. 1c). The patient was admitted to the intensive care unit with the impression of an acute coronary syndrome. Laboratory tests and an echocardiography were immediately arranged. The tests revealed a worsening of renal function (blood urea nitrogen $231 \mathrm{mg} / \mathrm{dL}$, creatinine $3.0 \mathrm{mg} / \mathrm{dL}$ ), a slight increase in the serum myoglobin $(123 \mathrm{ng} / \mathrm{mL})$, and the presence of hyperuricemia (696 $\mu \mathrm{mol} / \mathrm{L})$ and mild hyponatremia $(125 \mathrm{mEq} / \mathrm{L})$. The other tests were within the reference ranges. At echocardiography, moderately severe global hypokinesia (ejection fraction $\approx 38 \%$ ) with segmental hypokinesia of the midbasal portion of the inferolateral wall, and a circumferential pericardial effusion were found. Due to the possibility of aortic dissection, a transoesophageal echocardiogram was done, which excluded aortic dissection. While the patient was transferred to the closer "hub hospital" for an angiographic examination, he started to complain of severe abdominal pain with nausea and vomiting. Following a discussion between cardiologists and surgeons, the patient was then admitted for a computed tomography (CT scan) examination, which revealed the presence of free air in the mediastinum, mainly in the subcarinal, perioesophageal and paracardiac regions; a discrete pleural effusion with concomitant small pneumothorax in the left lung was also observed (Fig. 1d, e). A CT scan after water-soluble oral contrast revealed the presence of a perforation of the anterior wall of the oesophagus, about $6 \mathrm{~cm}$ above the cardiac region (Fig. 1f). The esophageal perforation was initially thought to be secondary to the transoesophageal echocardiography. However, after a careful examination of the CT scan, a "non identified foreign body" stuck through the 

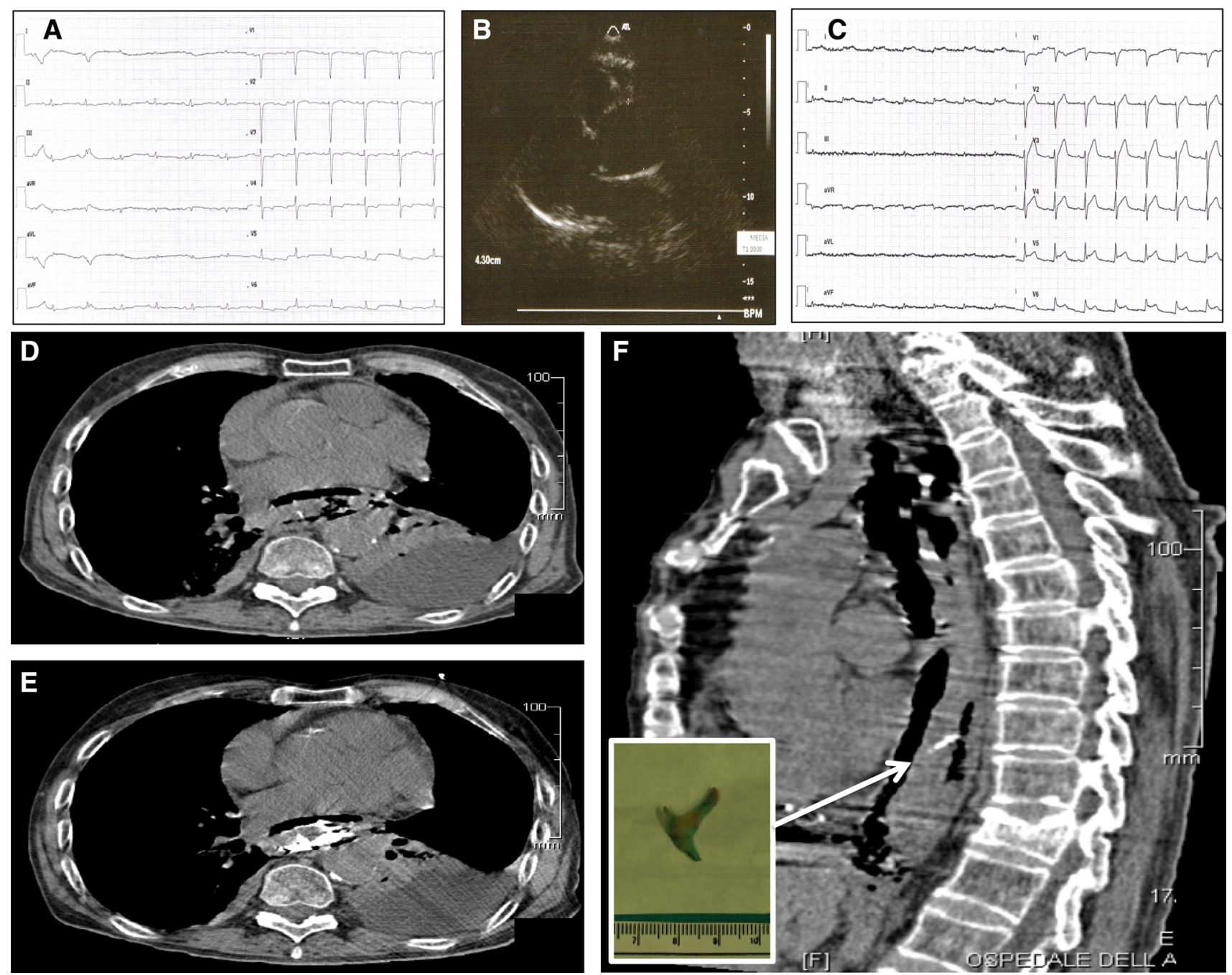

Fig. 1 ECG (a) and echocardiographic (b) findings at the first admission; ECG findings at the second admission (c); CT scan before (d, e) and after (f) administration of water-soluble oral contrast; inset in $\mathbf{f}$ shows the foreign body extracted

anterior wall of oesophagus was seen (Fig. 1f). The pleural effusion was found to be an empyema, and was drained. The patient then underwent surgical intervention to extract the foreign body, which was found to be a piece of bone (inset in Fig. 1f). When the doctor asked the wife what did they had eaten in the last few days, the wife reported: "Well, we had chicken, and my husband was almost strangled by a piece of bone!" After the intervention the patients were admitted to the intensive care unit, but his condition progressively worsened, and he died 6 weeks later.

Accidental ingestion of foreign bodies (mainly represented by fish bones and chicken bones), is frequent in adults [1]. As long as no occlusion or other complications develop, the clinical signs may be totally lacking. In spite of the mostly benign natural course, ingestion of foreign bodies is associated with an increased morbidity and mortality, mostly when the ingested foreign body is sharp or pointed [2]. The diagnosis of a foreign body ingestion is made primarily on the basis of the patient's medical history. In conclusion, this case highlights the atypical and misleading clinical presentation of this event that patients often underestimate and forget to report significant historical events to the doctor, causing delayed diagnosis and potentially an adverse clinical evolution.

Conflict of interest The authors declare that they have no conflict of interest.

Statement of human and animal rights All procedures performed in studies involving human participants were in accordance with the ethical standards of the institutional and/or national research committee and with the 1964 Helsinki declaration and its later amendments or comparable ethical standards.

Informed consent No informed consent. 


\section{References}

1. Chiu YH, Hou SK, Chen SC, How CK, Lam C, Kao WF et al (2012) Diagnosis and endoscopic management of upper gastrointestinal foreign bodies. Am J Med Sci 343:192-195
2. Loh KS, Tan LK, Smith JD, Yeoh KH, Dong F (2000) Complications of foreign bodies in the oesophagus. Otolaryngol Head Neck Surg 123(5):613-616 